\title{
Exploring the effects of 'fitspiration' and pressure from social media on body dissatisfaction and exercise motivation in male and female viewers in the UK
}

Body dissatisfaction is a recognised problem especially amongst women. There has been a significant rise in discussion concerning this topic since the existence of 'Fitspiration', a trend that is a combination of two words 'fitness' and 'inspiration'(1). 18 million posts were seen on Instagram when \#fitspiration was searched in $2018^{(2)}$. Exposure to fitspiration has been associated with adverse effects in social media users, such as increased risk of body dissatisfaction. Body dissatisfaction is an important issue as it is heavily associated with disordered eating, low self-esteem and suicidal thoughts. This research aimed to explore the impact of Fitspiration and pressure from social media on body dissatisfaction on various groups aged $18+$ years in the UK.

Participants were recruited via social media platforms and required to answer an online questionnaire which mostly consisted of Likert scales and included elements of two validated questionnaires designed to measure levels of body dissatisfaction. Ethical approval was granted from the university ethics committee and no funding was needed.

$\mathrm{n}=246$ respondents participated in the study. Descriptive and inferential statistics were used to analyse the data. Mann-Whitney U tests identified significant results at $(p=<0.05)$ suggesting that Fitspiration viewers exhibited greater body dissatisfaction, showing significantly more agreement "I worry about the way I look" ( $\mathrm{U}=4236.500, p=0.001)$. Those who felt pressurized from social media were more susceptible to body dissatisfaction and had higher agreement with the statements "I am ashamed of how I look" $(\mathrm{U}=3598.500, p=0.001)$ and "Preoccupied trying to change my body weight" $(\mathrm{U}=4044.000, p=0.001)$. Appearance was reported significantly more strongly as a motivator to exercise in fitspiration users compared to non-users of fitspiration users who were driven more by health concerns.

Despite positive intentions behind fitspiration to encourage a healthy lifestyle, this study suggests that motivation to exercise amongst fitspiration viewers was heavily associated with enhancing appearance compared to non-fitspiration viewers who reported to be more strongly motivated by health-related reasons. This is also one of the first studies to look into whether social media viewers experience pressure to change their appearance to look more 'attractive' and the results show that those who perceive pressure experience body dissatisfaction. This has given further insight in how White Papers such as Online Harms could take fitspiration content into consideration. The government must also implement the use of social media literacy in schools to target adolescents in order to prevent younger adults from experiencing negative effects from fitspiration, as upskilling will help individuals interpret images and messages from media correctly to combat body dissatisfaction ${ }^{(3)}$.

1. Tiggemann, M., \& Zaccardo, M. (2015). Body image, 15, 61-67.

2. Welker, K., Krysiuk, A., Philpot, S., Nabors, L., \& Goffena, J. (2019). The JSMS, 8, 2, 51-62.

3. Gordon, C. S., Rodgers, R. F., Slater, A. E., et al. (2020). Body Image, 33, $27-37$. 